\title{
Research on Intelligent Family Learning Assistant Based on Computer Vision Technology
}

\author{
Lunbin Li \\ Computer and Information Engineering \\ Heihe University \\ Heihe, China
}

\author{
Haikun Teng \\ Computer and Information Engineering \\ Heihe University \\ Heihe, China
}

\begin{abstract}
Because some students' learning habits are not good and their learning methods are not scientific, this leads to poor academic performance. In order to solve this problem, an intelligent home learning assistant software is developed on the basis of convolutional neural network is developed. It is learning assistant software which combines artificial intelligence, computer vision technology and auxiliary family education mode. No similar software has been found at home and abroad. The software can analyze the loopholes of students' learning knowledge points by recording the students' wrong questions, construct a unique picture of their mastery of personal knowledge, remind and supervise students to review the old and learn the new on time, and help students cultivate good learning habits by doing homework, so as to further improve students' academic performance. It has a certain society value.
\end{abstract}

Keywords-learning assistant; computer vision technology; artificial intelligence; convolution neural network

\section{INTRODUCTION}

College Entrance Examination is the biggest, most popular and fairest examination in China every year. It plays an important role in every parent and child's heart. Students and parents are trying their best to get a good result, but in this process, a very important problem has appeared, Why do some students achieve remarkable results while others do not achieve satisfactory results under the same educational resources and learning time input ${ }^{[1]}$ ? People from all walks of life have also expressed their opinions on this issue, but most people believe that it is caused by different learning habits and methods. Learning habits and learning methods are not formed overnight; students' needs have a good learning environment, correct guidance and strong self-discipline from an early age. However, this is not something everyone can have. Therefore, there is no good solution to this problem so $\mathrm{far}^{[2]}$. It's obviously unreasonable if students only rely more on teachers. The aim of this project is to help students develop good learning habits and improve their learning efficiency by combining artificial intelligence and computer vision technology with large data analysis.

\section{CURRENT SITUATION AT HOME AND ABROAD}

At present, there are all kinds of homework search software on the market, which are only used to search for answers, and

Teaching reform of Heihe University, Project number: xjg1729, project name: Construction and application of digital autonomous learning platform lack of innovation and humanization. This software is the first one in China that combines artificial intelligence, computer vision technology and auxiliary family education mode. The software can analyze the leaks of students' learning knowledge points by recording the students' wrong questions, construct a unique personal knowledge map for students, remind and supervise students to review the old and learn the new on time, and help students cultivate good learning habits by doing homework.

\section{PAIN POINTS TO BE SOLVED}

According to the students' daily routine, Xiao Wen, an assistant of intelligent phonetics, reminds and urges them to review the old and learn the new on time, so as to help them solve the problems of bad study habits and poor self-control.

This paper summarizes and sorts out the wrong and difficult problems in students' homework, establishes the students' exclusive wrong books, reminds students to review the wrong questions on time, and helps students form the good habit of "reading the wrong questions".

Improve students' self-awareness, enhance students' selfconfidence, and make learning more effective. Using big data to analyze, feedback and summarize the loopholes and difficult points of students' knowledge mastery in their homework, and to construct a unique knowledge network for each student so that students can clearly understand their shortcomings and remind students to solve and review on time, Let's learn to get twice the result with half the effort.

\section{SOLUTION}

To solve these problems, TickGo, intelligent home learning assistant software based on artificial intelligence and computer vision technology, is developed. The software is based on tensorflow and is written in Python language, and using the open source data model of tensorflow platform to train the project repeatedly, assistant learning guidance software is developed. The software can accurately identify and understand the meaning of the written text written by the user, correct and annotate the user's homework according to the data in the cloud server, set a unique "Xiaowen", that is, an assistant to help students review the old and learn the new, and take homework as a grasp to help students develop good learning habits, So as to improve academic performance. 


\section{PRINCIPLE OF RESEARCH AND DEVELOPMENT}

As we all know, any research and development of science and technology projects can't be separated from the corresponding scientific basis and theoretical support. Family learning assistant based on artificial intelligence and computer vision technology is based on convolution neural network theory, which is auxiliary learning guidance software.

Convolutional neural network is a kind of data neural network specially used to deal with similar network structure. It is a kind of deep learning. For example, time series data, image data. Nowadays, convolution network has become a research hotspot in the field of image understanding. Its weight sharing network structure makes it more similar to biological neural network, reduces the complexity of network model and reduces the number of weights. This advantage is more obvious when the network input is multi-dimensional image. The image can be directly used as the network input, avoiding the complex feature extraction and data reconstruction process in traditional recognition algorithm. Convolutional network is a multi-layer perceptron specially designed for recognizing twodimensional shapes. This network structure is invariant to translation, scaling and other forms of deformation. In a typical $\mathrm{CNN}$, the initial layers are usually alternating between the convolution layer and the undersampling layer, and the last layers near the output layer are usually fully connected networks.

\section{A. Training of Convolutional Neural Networks}

The main process is to learn the network parameters such as convolution kernel parameters of convolution layer and connection weights between layers. The prediction process is mainly based on input images and network parameters to calculate class labels ${ }^{[3]}$. The keys of convolutional neural network are network structure (including convolution layer, downsampling layer, full connection layer, etc.), back propagation algorithm and so on. The structure diagram of convolution neural network is shown in Fig. 1.

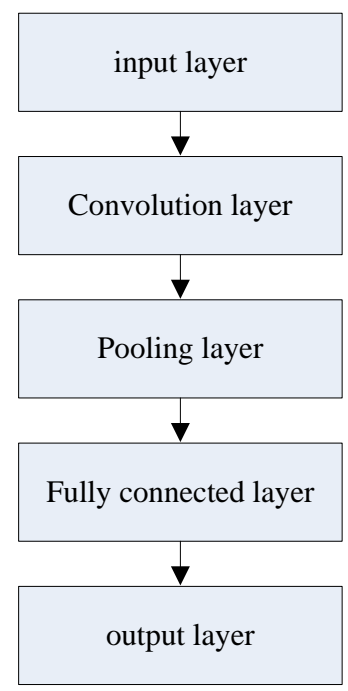

Fig. 1. The structure diagram of convolution neural network

\section{B. Convolution Layer}

Convolutional layer, each layer of convolutional neural network consists of several convolution units, and the parameters of each convolution unit are optimized by back propagation algorithm. The purpose of convolution operation is to extract different input features. The first layer of convolution may only extract some lower-level features such as edges, lines and angles. More layers of network can iteratively extract more complex features from lower-level features.

\section{Pooling Layer}

Pooling is another important concept in convolutional neural networks. It is actually a form of sampling reduction ${ }^{[4]}$. There are many different forms of nonlinear pooling functions, of which "Max pooling" is the most common. It divides the input image into several rectangular regions and outputs the maximum value to each sub region. Intuitively, this mechanism works because, after discovering a feature, its exact location is far less important than its relative location to other features. Pooling layer will continuously reduce the size of the data space, so the number of parameters and the amount of calculation will also decline, which to a certain extent also controls the over-fitting. In general, the convolution layer of $\mathrm{CNN}$ is periodically inserted into the pool layer.

\section{Fully Connected Layer}

In the CNN structure, one or more fully connected layers are connected after several convolution layers and pooling layers. Similar to MLP, each neuron in the fully connected layer is fully connected to all the neurons in the preceding layer. The fully connected layer can integrate the local information of the convolution layer or pooling layer which is classified and differentiated $^{[5]}$. In order to improve the performance of CNN network, the ReLU function is used to motivate each neuron in the whole link layer. The output value of the last fullconnection layer is passed to an output, which can be classified by soft Max logistic regression. This layer is also called soft Max layer. In general, CNN's full connection layer is the same as the MLP structure, and CNN's training algorithm also adopts BP algorithm.

\section{APPLICATION OF IMPROVED RECOGNITION TECHNOLOGY}

The improved face recognition technology based on machine vision is a computer vision engine based on AI depth learning convolution neural network and large data, The engine overthrows the traditional recognition methods based on spectral regression and matrix integrity constraints, adopts an improved face biometrics recognition technology based on machine vision, and uses a camera instead of a visual sensor to collect features of the face image, constructs a two-color reflection model of the face image, preprocesses the face image, and This paper combines machine vision with local difference binary model (LDBP) to improve face biometric recognition ${ }^{[6]}$. An intelligent home learning assistant based on artificial intelligence and computer vision technology applies this improved recognition technology. The improved recognition technology of face biometrics based on machine vision is shown in Fig. 2. 


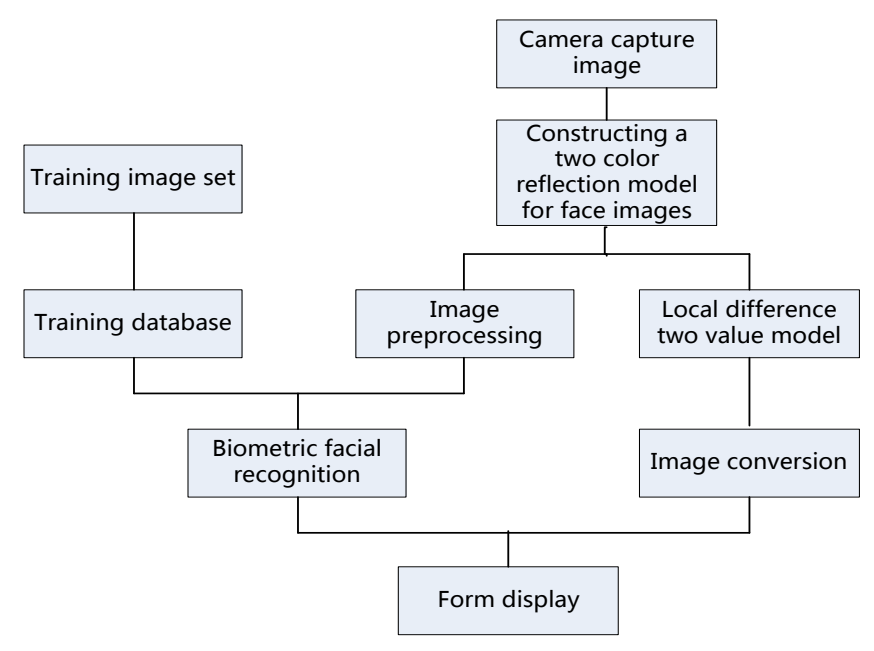

Fig. 2. An improved recognition technique for face biometrics.

\section{FUNCTIONAL DESIGN}

Monitor the students' daily homework, and feedback the results, warm tips on whether to meet the requirements, distance from the target. The teacher corrects students' homework and explains homework online. According to the students' homework errors, feedback analysis is conducted to form a unique online problem book for each student, which can help students learn from the past and correct mistakes in time. Using big data means to analyze feedback, summarize the leaks and doubtful difficulties of students' knowledge mastery, and build a unique knowledge network for each student, so that students can clearly understand their shortcomings.

\section{INNOVATION}

\section{A. Application Innovation}

With the rapid development of artificial intelligence technology, the application of artificial intelligence technology and new fields to achieve application innovation.

\section{B. Pattern Innovation}

The existence of the market is homework search software, only for search questions to find answers. Intelligent Family Learning Assistants analyze the leaks of students' learning knowledge points by recording students' wrong questions, construct a unique personal knowledge map for students, remind and supervise students to learn from the past and learn from the new on time, and help students cultivate good learning habits by doing homework, so as to further improve students' performance. Similar software has not been found at home and abroad.

\section{SOCIAL VALUE}

The social value of the project is mainly reflected in four aspects:

\section{A. Improving Students' Learning Efficiency}

Because the cultivation of learning habits and learning methods is not achieved overnight, students need a good learning environment, correct guidance and strong selfdiscipline from an early age, which is not everyone can have. However, after using the intelligent home learning assistant, students can learn efficiently in their spare time with the help of the assistant, develop good learning habits and improve learning efficiency.

\section{B. Optimizing Students' Learning Methods}

In the process of learning, students will always encounter different problems, encounter problems that can't be done, but through repeated practice to gradually familiarize themselves with the practice of such topics, but the deep-seated problems are always ignored, students may encounter problems that can't be done because a certain knowledge point to master not solid, But students spend a lot of time doing repetitive tasks. Now the intelligent home learning assistant can sum up and sort out the wrong questions and difficult problems in the students' homework to form the wrong question book, analyze feedback and summarize the loopholes and doubtful difficulties of students' knowledge mastery by means of big data, and construct a unique knowledge network for each student's knowledge mastery. Let students clearly understand their own shortcomings, and timely remind students to solve and review to grasp the weak points of knowledge, optimize the learning methods.

\section{Enhance Students'self-confidence}

Through the construction of a unique knowledge network for students, so that students more clearly understand their lack of knowledge, for their own better formulation of learning routes, to improve learning efficiency, thus improving the results of students, improve their self-confidence will be significantly improved, the physical and mental development of students are greater benefits.

\section{Optimizing Competition Mode}

When students have a clearer understanding of their strengths and weaknesses, they will pay more attention to selfoptimization and improvement, to achieve a "self-competition" state, in order to reduce the negative impact and vicious competition caused by competition between students.

\section{CONCLUSION}

Under the high competitive situation of China's college entrance examination and the tide of vigorous development of artificial intelligence, the use of artificial intelligence technology to develop a system to help students cultivate good learning habits and improve student performance can't only help students and parents reduce the burden and pressure on the micro level, but also optimize social competition on the macro level, that is, from blindly everyone into self-optimization model competition. It is a great help to train high-quality talents.

Based on convolution neural network, artificial intelligence and computer vision technology, and combined with large- 
scale data analysis, an assistant intelligent home learning assistant software is designed. In the design process, the pain points that need to be solved are analyzed and solutions are put forward.

The application of artificial intelligence technology in the field of family-assisted education system to construct a new model is the first case in China, which realizes model innovation and has certain social value.

\section{REFERENCES}

[1] Rao Hao, Aided learning App design based on Android. Computer Knowledge and Technology, 2018.(In Chinese)

[2] Luyao Pu, Based on Android's "work-study reminder assistant" APP development. Science and technology innovation, 2018. (In Chinese)

[3] Tao Zou, Intelligent Knowledgeable Vehicle Assistant Based on CNN Model of Deep Learning. Computer Knowledge and Technology, 2018. (In Chinese)

[4] Jucheng Yang, Na Liu, Shanshan Fang, et al.Review of face recognition methods based on deep learning. Journal of Tianjing University of Science\&Technology, 2016, 31(6), pp.1-10. (In Chinese)

[5] Xiao Ma, Fandong Zhang, Jufu Feng, Sparse representation via deep learning features based face recognition method. CAAI transactions on intelligent systems, 2016, 11 (3), pp.279-286. (In Chinese)

[6] Tao Han. Exp ressive exp ression mapping with ratio images of machine vision, Science technology and engineering, 2017, 17(14), pp.239-242. (In Chinese) 